\title{
Maximum number of total born piglets in a parity and individual ranges in litter size expressed as specific characteristics of sows

\author{
Gertraude Freyer
}

\begin{abstract}
Background: The objective of this study was to underline that litter size as a key trait of sows needs new parameters to be evaluated and to target an individual optimum. Large individual variation in litter size affects both production and piglet's survival and health negatively. Therefore, two new traits were suggested and analyzed. Two data sets on 5509 purebred German Landrace sows and 3926 Large White and crossing sows including at least two parental generations and at least five parities were subjected to variance components analysis.

Results: The new traits for evaluating litter size were derived from the individual numbers of total born piglets (TBP) per parity: In most cases, sows reach their maximum litter size in their fourth parity. Therefore, data from at least five parities were included. The first observable maximum and minimum of TBP, and the individual variation expressed by the range were targeted. Maximum of TBP being an observable trait in pig breeding and management yielded clearly higher heritability estimates $\left(h^{2} \sim 0.3\right)$ than those estimates predominantly reported so far. Maximum TBP gets closer to the genetic capacity for litter size than other litter traits. Minimum of TBP is positively correlated with the range of TBP $\left(r_{p}=0.48, r_{g}>0.6\right)$. The correlation between maximum of TBP and its individually reached frequency was negative in both data sets $\left(r_{p}=-0.28\right.$ and -0.22 , respectively). Estimated heritability coefficients for the range of TBP comprised a span of $h^{2}=0.06$ to 0.10 .
\end{abstract}

Conclusion: An optimum both for maximum and range of total born piglets in selecting sows is a way contributing to homogenous litters in order to improving the animal-related conditions both for piglets' welfare and economic management in pig.

Keywords: Pig, Litter size, Individual maximum, Variation, Heritability, Trait correlation

\section{Background}

In pig breeding, the number of piglets born alive per parity (NBA) is a trait of economically importance, but affected by multiple factors [1]. Heritability is always reported to be relatively low, and the wise breeding decision is an enduring challenge. Many biologic effects on litter size emphasize the problem: healthy conditions of the sow, specific effects due to the birth process, individual maternal influences. Multifold environmental impacts do play a role as well. One specific example on intrinsic effects is the content of serum immunoglobulin being lower in piglets from larger litters [2].

Correspondence: gertraude.freyer@uni-rostock.de

Leibniz Institute of Farm Animal Biology (FBN), Institute for Genetics and Biometry, Wilhelm-Stahl-Allee 2, D-18196 Dummerstorf, Germany
Heritability of total number of piglets born per litter (TBP) is larger than that of NBA being more sensitive against several long and short-term effects. But no heritability estimates $>0.19$ have been found in such study reports, neither for TBP and nor for NBA. Genetic variation between and within breeds was triggering substantial genetic increase in prolificacy in recent years [3], accompanied by increasing TBP and NBA. However, correlation coefficients of individual litter sizes were $<0.75$ [4]. On the other hand, there is much variation in these traits that can be exploited in further breeding decisions. A low variation in individual prolificacy across parities could minimize negative side effects both on survival of piglets and on management in commercial swine production. Less work would be needed for managing sows having an almost constant TBP, ideally being equal to NBA, through

(C) The Author(s). 2018 Open Access This article is distributed under the terms of the Creative Commons Attribution 4.0 International License (http://creativecommons.org/licenses/by/4.0/), which permits unrestricted use, distribution, and 
all their parities. Nursing and feeding conditions could be stabilized. A reduced individual variation in TBP would help to improving efficiency of piglet production and animal welfare. Targeting an individual optimum in litter size is presumably a thorny issue, but worth investigating.

The objective of this study was on the genetic capacity of TBP expressed by the individual maximum TBP (Max_TBP) and on the individual variation in TBP (Range_TBP). Two data sets based on genetically different pools from commercial swine production in Germany were subjected to analyzing phenotypic trait observations by means of a simple but widely used and recognized methodical approach in order to estimate heritability coefficients for these new parameters for litter traits.

\section{Material and Methods}

\section{Phenotypic data}

Fecundity data collected in 30 farms from January 1997 through June 2010 was provided by the Hybridschweinezuchtverband Nord/Ost e.V. Malchin, Germany. In the first data set, 15,271 German Landrace sows (RA01) stemming from 1425 sires belonged to about 800 contemporarily farrowing groups. Heritability coefficients on this large dataset based on first parities have already been reported $\left(h^{2}=0.10\right.$ and 0.13 for NBA respective TBP) [5]. In the present study, 5509 sows with at least five parities were considered (Table 1). Most phenotypic

Table 1 Basic statistics on the investigated traits individual maximum and minimum of number total born piglets (Max_TBP and Min_TBP), and individual range of number total born piglets (Range_TBP), number of total born piglets and number of piglets born alive in first parity (TBP_first and NBA_first) in German Landrace sows (RA01) and Large White sows including crossing sows (RACOM) with at least five individual parities each

\begin{tabular}{|c|c|c|c|c|}
\hline & \multicolumn{4}{|c|}{ Overall observed } \\
\hline & Mean & $\begin{array}{l}\text { Standard } \\
\text { deviation }\end{array}$ & Minimum & Maximum \\
\hline \multicolumn{5}{|c|}{ German Landrace sows (RA01, $n=5509$ ) } \\
\hline Min_TBP & 8.67 & 2.581 & 1 & 17 \\
\hline Max_TBP & 15.46 & 2.181 & 9 & 29 \\
\hline Range_TBP & 7.21 & 2.908 & 1 & 22 \\
\hline TBP_first & 11.46 & 2.850 & 1 & 21 \\
\hline NBA_first & 10.81 & 2.739 & 1 & 20 \\
\hline Overall parity number & 6.57 & 1.480 & 5 & 15 \\
\hline \multicolumn{5}{|c|}{ Large White and crossing sows (RACOM, $n=3926)$} \\
\hline Min_TBP & 9.34 & 2.700 & 1 & 17 \\
\hline Max_TBP & 15.63 & 2.260 & 8 & 26 \\
\hline Range_TBP & 6.29 & 2.803 & 1 & 22 \\
\hline TBP_first & 11.53 & 2.905 & 2 & 20 \\
\hline NBA_first & 10.84 & 2.779 & 1 & 20 \\
\hline Overall parity number & 6.90 & 1.830 & 5 & 16 \\
\hline
\end{tabular}

individual maximum total born piglets of sows occurred in their fourth parities (Fig. 1). This suggests that the true individual potential denoted by Max_TBP mostly is observable when the sow has finished at least four parities. The pedigree contained 30,620 individuals in total including 6168 base animals and two or more parental generations of the recorded sows in RA01. The second data set (RACOM) meeting the same conditions as above comprised 3926 German Large White purebred sows and sows from crossings with German Landrace, stemming from 436 sires (details on crossing sows: dam was Large White for 211 sows, sire was Large White for 2053 sows). Therefore, three breed groups had to be allowed for in RACOM. The whole pedigree was larger in RACOM, containing 37,003 individuals with 6567 base animals. Inbreeding was not an issue, neither in RA01 and nor in RACOM.

\section{Description of investigated traits and influencing factors}

The target trait was the individual maximum of TBP (Max_TBP). As a secondary trait, a characteristic for describing individual variation in TBP of sows should be easily observable in swine production was on focus. Range_TBP was used here on the basis of individual Max_TBP and individual minimum TBP (Min_TBP). A sufficient number of individual parities of each sow $\mathbf{h}$ was necessary to calculate

$$
\text { Range } \mathrm{TBP}_{\mathrm{hk}}=\mathrm{Max}_{-} \mathrm{TBP}_{\mathrm{hi}}-\mathrm{Min}_{-} \mathrm{TBP}_{\mathrm{hj}} \text {, }
$$

where $\mathbf{i}$ and $\mathbf{j}$ denoted different parity numbers yielding the single maximum respective minimum of TBP and $\mathbf{k}$ was the number of individually available parities.

Min_TBP and Max_TBP were in fact new traits based on their first individual occurrence. Range_TBP describes a specific situation that cannot be expressed by original measurements. Calculating Range_TBP is therefore comparable to the blood pressure amplitude in medicine. Minimum and maximum were obtained from different parities of a single sow. E.g., the specific parity number and contemporarily group of sows (expressed by herd, year and season of parity) leading to the specific trait observation was considered as fixed effect on Max_TBP and Min_TBP. A peculiarity was that the year of first farrowing had a significant effect on Max TBP $(P<0.0001)$ suggesting a targeted selection effect. In Range_TBP, the situation was different in terms that significance of fixed effects was found for herd, year and season related to Min_TBP. Further, the number of available parities (in analyses of using greater than five parities) resulted in a significant effect on Range_TBP, but not the parity number related to Min_TBP or Max_TBP. 


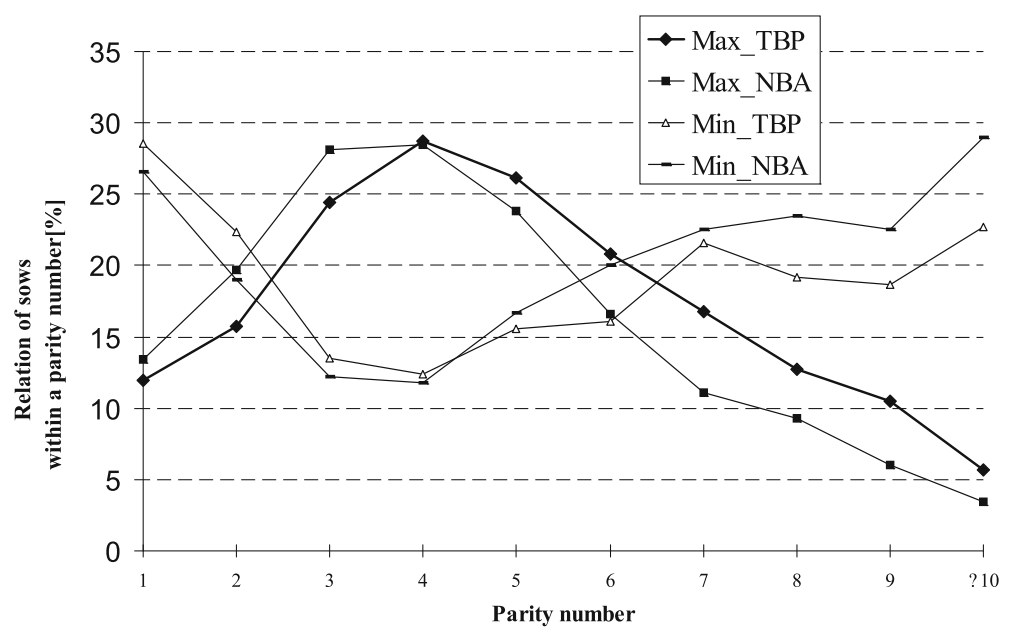

Fig. 1 Percentage of sows performing their individual maximum and minimum number of total born piglets (Max_TBP and Min_TBP) and number of piglets born alive (Max_NBA and Min_NBA) in a parity number related to the total number of sows in this parity group (denoted by the horizontal axis). Both unique and multifold occurrences of individual extremes were included in this diagram obtained from 5509 German landrace sows (RA01) with 36,894 phenotypic records in total

All significant effects were allowed for trait specifically in statistical analyses. For all targeted traits, skewness and kurtosis were negligibly small. The precondition of a normal distribution was fulfilled.

NBA at first parity (NBA_first) has apparently been the most respected trait for production purposes so far as suggested from the majority of relevant reports. Therefore, it was contemplated in parallel throughout the study.

\section{Statistical analyses}

Genetic parameters of the investigated traits were estimated on the basis of the animal model

$$
\mathbf{y}=\mathbf{X b}+\mathbf{Z u}+\mathbf{e}
$$

where $\mathbf{y}$ is the vector of trait observations (phenotypes), $\mathbf{b}$ vector of fixed effects (breed code, farm, herd-group, year and season of parity, actual parity number (see also above for further significant effects)), $\mathbf{u}$ vector of direct additive genetic (animal) effects, and $\mathbf{e}$ the vector of residual effects. $\mathbf{X}$ denotes the incidence matrix of fixed effects, $\mathbf{Z}$ matrix of direct additive-genetic effects. $\mathbf{u}$ and $\mathbf{e}$ were normally distributed $(0, \mathbf{A} \otimes \mathbf{G})$ with numerator relationship matrix $\mathbf{A}$ and additive genetic variance-covariance structure $\mathbf{G}$, and $\left(0, \mathbf{I}_{\mathrm{e}} \otimes \mathbf{R}\right)$ for $\mathbf{e}$, respectively, with. $\mathbf{I}_{\mathrm{e}}$ identity matrix and $\mathbf{R}$ variance-covariance structure of residuals. The symbol $\otimes$ denotes the Kronecker product.

The variance components analyses aiming at estimating heritability coefficients and their standard errors (SE) were carried out by means of univariate modeling and based on one individual trait observation/trait value each for a single sow. Computer programs VCE 5.1.2 and PEST V4.0 were used for the estimating heritability (and SE) based on
REML and were run on a LINUX machine $[6,7]$. SAS package [SAS Institute Inc., Cary, NC, US, versions 9.2 and 9.4] was used for general statistics including Pearson correlation coefficients (accompanied by the error probability P) and for testing fixed effects and group differences by means of GLM and MIXED procedures.

Min_TBP and Max_TBP were considered once as unique trait values at their first occurrence. A repeatability model was therefore not applied for any of the traits.

\section{Results}

\section{Heritability estimates}

The relative additive variance components representing the heritability coefficients are shown for both samples (Table 2). In fact, results of two analyses per sample are listed: (i) results from analyses based on all parities $\geq$ five, and (ii) results based on exactly five parities. Heritability estimates of Max_TBP were within a span of 0.29 to 0.39 . Standard errors were low in both samples $(\mathrm{SE}=0.02 \ldots 0$. 04). Heritability estimates for Min_TBP were considerably lower, namely $h^{2}=0.07 \ldots 0.11$ (in most cases, $\mathrm{SE}<0.02$ ).

Heritability estimates of Range_TBP were lowest in RA01 $\left(h^{2}=0.06 \pm 0.01\right)$ compared to RACOM $\left(h^{2}=0.10 \pm 0.02\right)$, whereas the use of exactly five parities let to closer results between both samples, but accompanied by larger SE (Table 2).

\section{Frequency of individual maxima and minima in contemplation to Range_TBP}

About 26 percent of sows reached their Max_TBP more than once during their available parities. Three to four percent yielded the same maximum more than twice. The results were almost equal for RA01 and RACOM. 
Table 2 Estimates of relative additive genetic variance components expressing the heritability coefficients $\left(h^{2}\right)$ and their standard errors $\left(\mathrm{VC}_{\text {rel }} \pm \mathrm{SE}\right)$ on the investigated traits ${ }^{\mathrm{a}}$ Min_TBP, Max_TBP and Range_TBP and in addition on NBA in first parity based on univariate analyses

\begin{tabular}{|c|c|}
\hline \multirow[t]{2}{*}{ Trait } & $\begin{array}{l}\text { Estimated variance components in relation } \\
\text { to the total phenotypic variance }\end{array}$ \\
\hline & Additive genetic \\
\hline
\end{tabular}

RA01 (German Landrace sows, $n=5509$ )

(i) information on five or more parities used for estimation

$\begin{array}{lll}\text { Min_TBP } & 0.112 \pm 0.014 & 0.888 \pm 0.014\end{array}$

$\begin{array}{lll}\text { Max_TBP } & 0.298 \pm 0.021 & 0.702 \pm 0.021\end{array}$

$\begin{array}{lll}\text { Range_TBP } & 0.056 \pm 0.011 & 0.944 \pm 0.011\end{array}$

NBA_first $\quad 0.123 \pm 0.017$

$0.877 \pm 0.017$

(ii) information on exactly five parities used for estimation

$\begin{array}{lll}\text { Min_TBP } & 0.070 \pm 0.015 & 0.930 \pm 0.015 \\ \text { Max_TBP } & 0.283 \pm 0.023 & 0.717 \pm 0.023 \\ \text { Range_TBP } & 0.066 \pm 0.012 & 9.934 \pm 0.012\end{array}$

RACOM (Large White and crossing sows, $n=3926$ )

(i) information on five or more used for estimation

$\begin{array}{lll}\text { Min_TBP } & 0.098 \pm 0.018 & 0.902 \pm 0.018 \\ \text { Max_TBP } & 0.373 \pm 0.030 & 0.627 \pm 0.030 \\ \text { Range_TBP } & 0.104 \pm 0.022 & 0.896 \pm 0.022 \\ \text { NBA_first } & 0.116 \pm 0.020 & 0.884 \pm 0.020\end{array}$

(ii) information on exactly five parities used for estimation

$\begin{array}{lll}\text { Min_TBP } & 0.070 \pm 0.024 & 0.930 \pm 0.024\end{array}$

$\begin{array}{lll}\text { Max_TBP } & 0.390 \pm 0.040 & 0.610 \pm 0.040\end{array}$

Range_TBP $\quad 0.084 \pm 0.026 \quad 0.916 \pm 0.026$

${ }_{\text {investigated traits were individual maximum and minimum of number total }}$ born piglets (Max TBP, Min_TBP), and individual variability in number total born piglets (Range_TBP); number of piglets born alive in first parity (NBA_first) for comparing to heritability estimates from earlier studies

The relation was less for Min_TBP. 20 percent of sows showed their Min_TBP twice or more.

Negative phenotypic correlation coefficients being very similar in both samples were estimated for the individual frequency of Max_TBP and Range_TBP $\left(r_{p}=-0.25\right.$ and-0.23, $P<0.0001)$. The even stronger negative correlation of individual frequency of Min_TBP and Range_ TBP was almost the same in RA01 and RACOM $\left(r_{p}=-0.32\right.$ and $\left.-0.31, P<0.0001\right)$.

In most cases of RA01, multiple maxima occurred for Max_TBP $=13 \ldots 16$ piglets (in 996 cases, respective 69 percent of sows with multiple maxima). In RACOM, the "preferred span of maxima" was Max_TBP $=13 \ldots 17$ piglets. Multiple minima were observed most frequently for Min_TBP $=9 \ldots 11$ in RA01 (in 56 percent of sows with multiple minima), and $\mathrm{Min} \_\mathrm{TBP}=10 \ldots 12$ in RACOM. In total, RACOM was more homogenous in Range_TBP, confirmed by lower mean and lower standard deviation (Table 1).

\section{Phenotypic and genetic trait correlations}

Almost all estimated trait phenotypic correlation coefficients were significant (Table 3). The estimated genetic correlation coefficients were highly positive for Max TBP and Range_TBP: $r_{g}=0.617 \pm 0.061$ in RA01 and $r_{g}=0.761 \pm 0.067$ in RACOM. The genetic correlation of Range_TBP and Min_TBP were not significant, $r_{g}=0.141 \pm$ 0.132 in RA01 and $r_{g}=0.214 \pm 0.180$ in RACOM.

Phenotypic correlation coefficients for piglets born alive in first parity (NBA_first) and Range_TBP were significantly negative $\left(r_{p}=-0.15\right.$ in RA01 and $r_{p}=-0.22$ in RACOM, Table 3). This seems advantageous when focusing on a very early breeding decision. However, positive genetic correlations of NBA_first and Range_TBP minimize the expectations $\left(r_{g}=0.33\right.$ in RA01 and $r_{g}=0.31$ in $\mathrm{RACOM}, \mathrm{SE}=0.09$ and 0.10 , respectively).

\section{Discussion}

In commercial swine production, litter size is a key trait. However, individual numbers of total born and piglets born alive vary in subsequent parities. So far, this fact has hardly been targeted as a special parameter of sows. In this study, individual ranges of TBP were chosen to do so as a simple trait in order to draw attention to this problem and to suggest coping with.

Selection on larger TBP increases NBA but in many cases it also increases the number of still born piglets. For this reason and to respecting the individual feeding capacity of a sow, searching for an individual optimum TBP should be a target in pig breeding. In the investigated material, a clear trend of increasing mean performances in TBP and NBA of first parity were observed during 1997 to 2008. At the same time, there was no increase in mean Range_TBP, but standard deviations of Range_TBP showed an increased trend from 1997 to 2008 very clearly (not shown in further detail). This suggests potential for respecting individual variation without necessarily decreasing litter sizes on average.

Respecting individual Max_TBP and individual Range_ TBP could be a way for optimizing the management process in swine production and improving animal welfare directly on the sow's level. Litter size traits in pigs are likely more affected by maternal than by paternal components. One might argue that the number of five individual parities to evaluate the genetic capacity of TBP and individual variation is a challenging limitation. The preference on fast selection decisions in sows focuses on their performances in NBA_first.

Which parameter really matters for evaluating a sow for litter size with respect to efficiency? Observed Max_TBP is an individual parameter for the genetic capacity of litter size as clearly suggested by larger heritability estimates compared to traditionally used litter size traits (Table 2). 
Table 3 Phenotypic correlation coefficients of individual variability in number total born piglets (Range_TBP), maximum and minimum number of total born piglets (TBP_Max and TBP_Min), and in addition both the number piglets born alive in first parity (NBA_first) and the individual relative number of still born piglets (SB_rel) ${ }^{a}$ obtained from RA01 ( $n=5509$, above diagonal) and from RACOM ( $n=3926$, below diagonal), Pearson correlation coefficients were significant at $P<0.0001$, besides those marked by superscript letters

\begin{tabular}{|c|c|c|c|c|c|c|}
\hline & \multicolumn{6}{|c|}{ RA01 (above diagonal) } \\
\hline & & Range_TBP & Max_TBP & Min_TBP & NBA_first & SB_rel \\
\hline RACOM & Range_TBP & -——- & 0.478 & -0.669 & -0.145 & 0.079 \\
\hline \multirow[t]{4}{*}{ (below diagonal) } & Max_TBP & 0.475 & -—-二 & 0.316 & 0.277 & 0.287 \\
\hline & Min_TBP & -0.672 & 0.332 & - - - - & 0.392 & 0.159 \\
\hline & NBA_first & -0.219 & 0.257 & 0.452 & - - - & $0.011^{\mathrm{b}}$ \\
\hline & SB_rel & 0.086 & 0.286 & 0.148 & $-0.003^{c}$ & -— \\
\hline
\end{tabular}

${ }^{\mathrm{a}} \mathrm{SB}$ rel was based on phenotypic on all available parities observations on still births adjusted for fixed effects of breed indicator (within RACOM), parity number, herd, year and season using the basic data set (in total, 63,000 records in RA01, 41,500 records in RACOM) for all sows included in the current study

${ }^{\mathrm{b}} P=0.4256$

${ }^{c} P=0.8437$

Targeting an optimum TBP does not mean selecting for highest Max_TBP. This trait should be evaluated in order to find a basis for choosing the optimum. Simultaneously, a small individual Range_TBP should be preferred in future breeding objectives. Comparing single sows yielding extremely different Range_TBP reveals the practical value of low individual variation in litter size. Sows with a Max TBP of 13 to 16 showed the lowest Range_TBP and simultaneously the most repeatable Max_TBP, accompanied by fewest still births. The significant connection between still births and Range_TBP is also shown by dividing sows into range classes and by phenotypic correlations (Fig. 2, Table 3 ) and by means of eight single sows being extremely different in their Range_TBP (Table 4).

In the present study, the relative genetic variance (heritability) estimated for Range_TBP was about six to 10 percent. RACOM was more homogenous in Range_TBP than RA01. Due to the large part of crossing sows included in RACOM, this could be a response of favorable non-additive components triggering more stabilizing factors on expressing these traits. On the other hand, despite fewer sows with trait observations, the pedigree was much larger for RACOM than for RA01, a fact that could have caused higher heritability estimates.

Selecting sows with an individual optimum TBP respective NBA for breeding could contribute to more phenotypically (and especially environmentally) balanced sustainability. Priority should be given to feeding capacity and maternal behavior.

Birth weight of each single piglet could likely be an additional co-variable in evaluating litter size traits [8]. However, such data was not available for this study.

Table 4 Single sows from RACOM with exactly five parities and extremely low or extremely large ranges of their number in total born piglets per parity (Range_TBP), maximum number of total born piglets (Max_TBP) marked in bold, and the related absolute number of still born piglets (SB)

\begin{tabular}{|c|c|c|c|c|c|c|c|c|c|c|c|c|c|}
\hline \multirow{3}{*}{$\begin{array}{l}\text { group with respect to } \\
\text { Range_TBP and breed }\end{array}$} & \multirow[b]{3}{*}{ ID } & \multicolumn{10}{|c|}{ Parity number } & & \\
\hline & & \multicolumn{2}{|l|}{1} & \multicolumn{2}{|l|}{2} & \multicolumn{2}{|l|}{3} & \multicolumn{2}{|l|}{4} & \multicolumn{2}{|l|}{5} & \multicolumn{2}{|c|}{ Total } \\
\hline & & TBP & SB & TBP & $S B$ & TBP & SB & TBP & SB & $\mathrm{TBP}$ & $S B$ & TBP & SB \\
\hline \multicolumn{14}{|l|}{ Low Range_TBP } \\
\hline Crossing sow & 196679 & 16 & 2 & 15 & 0 & 16 & 0 & 15 & 0 & 15 & 0 & 77 & 2 \\
\hline Large White & 285278 & 13 & 2 & 13 & 1 & 13 & 1 & 14 & 1 & 13 & 0 & 66 & 5 \\
\hline Large White & 106820 & 11 & 0 & 12 & 0 & 12 & 0 & 12 & 0 & 11 & 0 & 58 & 0 \\
\hline Crossing sow & 200791 & 10 & 0 & 10 & 1 & 10 & 0 & 11 & 1 & 11 & 0 & 52 & 2 \\
\hline \multicolumn{14}{|l|}{ Large Range_TBP } \\
\hline Large White & 106324 & 17 & 5 & 20 & 2 & 18 & 6 & 7 & 0 & 5 & 0 & 67 & 13 \\
\hline Crossing sow & 194908 & 10 & 0 & 17 & 2 & 18 & 0 & 17 & 4 & 1 & 0 & 63 & 6 \\
\hline Large White & 107757 & 19 & 0 & 18 & 1 & 17 & 1 & 4 & 0 & 13 & 0 & 71 & 2 \\
\hline Large White & 272177 & 9 & 0 & 11 & 0 & 14 & 2 & 26 & 13 & 14 & 1 & 74 & 16 \\
\hline
\end{tabular}



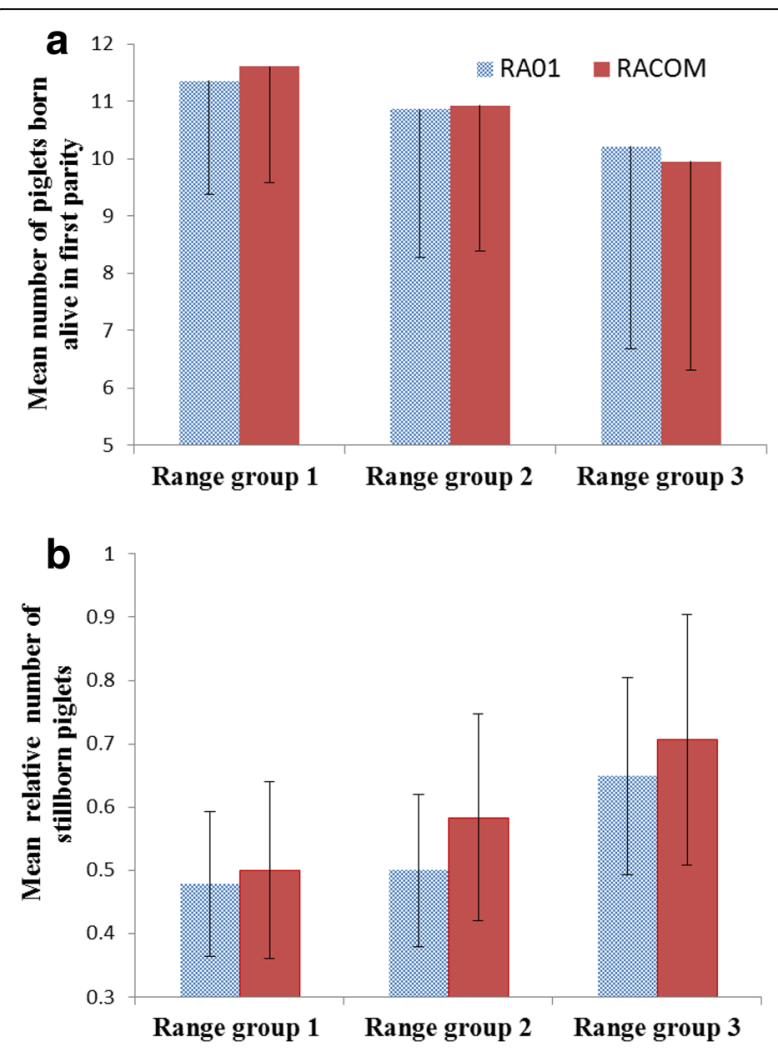

Fig. 2 Phenotypic means of piglets born alive in first parity (a) and relative mean of still born piglets based on phenotypic observations adjusted for fixed effects of breed indicator, litter number, herd, year and season (b) for all sows included in the current study (5509 sows in RA01, 3926 in RACOM), grouped by their ranges of total born piglets: Low ranges (difference between individual maximum and minimum number of total born piglets is 1 to 4 in Range group 1), medium ranges (5 to 9 in Range group 2) and large ranges ( $\leq 10$ in Range group 3), respectively. Significance of differences at $P<0.0001$ in (b) only: Range groups 1: 3 and Range groups 2: 3 in RA01, Range groups 1: 3 in RACOM

NBA in first parity was analyzed in parallel resulting in very similar heritability estimates as reported before [5]. A selection effect on sows under study was therefore not suspected.

No earlier reports based on individual maxima and on individual female variation of TBP have been found in the literature related to pig breeding. A recent study carried out in the Netherlands was based on boars' observations through their daughters [9]. For a deeper insight into the genetics of litter size and related traits, SNP technology is being widely adapted. Such studies have been reviewed [10], and candidate genes of reproduction traits in sows were reported based on different pig populations, partly with contradictory results [11-13].

Non-additive effects of single candidate genes (or chromosomal segments) interacting with others could play a different role for TBP and NBA in first and higher parities [11]. Therefore, patterns of age-dependently (inter-) acting genes, and genes affecting environmental sensitivity, could also affect the individual variation of traits related to litter size. Two candidate genes contributing to variation in TBP were reported [8]. One of them is involved in buffering environmental and genetic factors. From the same study, a genetic correlation between TBP and its variation based on boars' breeding values was reported $\left(r_{g}=0\right.$. 49 [8]). This was very similar to the phenotypic correlation of Range_TBP and Max_TBP in the present study $\left(r_{p}=0.48\right.$, Table 3$)$.

The results of the study reported here suggest that an individual optimum number of total born and alive born piglets per parity of a sow can be found by respecting individual Max_TBP and Range_TBP. Heritability of sows' individual Max_TBP is higher than heritability estimates for litter size traits as published so far. More detailed investigations on the basis of a genome wide association study and surely using methods allowing for genotype by environmental interactions could lead to increasing the knowledge on the responsible genes, multifold induced interactions and their functions. Focusing on sows being extremely different in their individual variation in litter size would be of value in this continuing research process.

\section{Conclusions}

Using the individual maximum number of total born piglets as a new trait in genetic analyses reveals considerably higher heritability estimates $\left(h^{2} \sim 0.3\right)$ than those from using ordinary litter size data repeatedly reported before. Information on the individual maximum is more suited to reflect the genetic proliferative potential of the sow. Many sows reached their maximum of 13 to 16 piglets in a parity more than once. Therefore, this trait is suggested to find an individual optimum litter size for improving both the management process and animal's health. A sufficient evaluation of sows regarding both their individual capacity in litter size and the related individual variation (e.g. range as a simple secondary trait) is possible if data of at least five parities is available. Individual range of litter size in sows has a heritable component $\left(h^{2}=0.06\right.$ to 0.10$)$.

\section{Abbreviations}

Max_NBA: Individual maximum number of piglets born alive related to a specific parity; Max_TBP: Individual maximum number of total born piglets of a sow related to a specific parity; Min_NBA: Individual minimum number of piglets born alive related to a specific parity; Min_TBP: Individual minimum number of total born piglets of a sow related to a specific parity;

NBA: Individual number of piglets born alive per parity; NBA_first: Individual number of piglets born alive in first parity; P: Error probability; RA01: Data set from German Landrace; RACOM: Data set from Large White and crossing sows; Range_TBP: Individual range of number of total born piglets of a sow (individual variation); SB: Number of still born piglets of a sow;

SB_rel: Individual number of still born piglets in a parity adjusted for fixed effects of herd, year and season of farrowing; SE: Standard error; TBP: Total number of piglets per parity; VCE rel: Relative variance component; Further, symbols on population genetic parameters were used: $h^{2}$ - heritability coefficient; $r_{g}$ and $r_{p}$ - genetic and phenotypic correlation coefficients. Other specific symbols were used only once in the methods section (with explanation) 


\section{Acknowledgements}

Data used in this study was provided by Hybridschweinezuchtverband Nord/Ost e.V. Malchin, Germany. Thanks to Dipl.-Ing. Renate Schuster and Dr. Sigfried Hoffmann for valuable communications.

\section{Funding}

No funding from a third party.

\section{Availability of data and materials}

Not applicable; data originated from a commercial pig breeding company.

\section{Authors' contributions}

The author read and approved the final manuscript.

\section{Ethics approval}

Not applicable.

\section{Competing interests}

The author declares that she has no competing interests.

\section{Publisher's Note}

Springer Nature remains neutral with regard to jurisdictional claims in published maps and institutional affiliations.

Received: 25 July 2017 Accepted: 1 May 2018

Published online: 02 July 2018

\section{References}

1. Lawlor PG, Lynch PB. A review of factors influencing litter size in Irish sows. Irish Veterinary J. 2007;60:359-66.

2. Nguyen K, Cassar G, Friendship RM, Dewey C, Farzan A, Kirkwood RN. An investigation of induced parturition, birth weight, birth order, litter size, and sow parity on piglet serum concentrations of immunoglobulin G. J Swine Health Prod. 2013;21(3):139-43.

3. Tribout T, Caritez JC, Gogué J, Gruand J, Billon Y, Dividich JLE, Quesnel H, Bidanel JP. Estimation of realized genetic trend in French large white pigs from 1977 to 1998 using frozen semen. J de la Rech Porcine en France. 2003;35:85-292.

4. Barbosa L, Lopes PS, Regazzi AJ, Torres RD, Santana JR, Veroneze R. Estimation of genetic parameters for litter size in pigs using multi-trait analyses. Revista Brasileira Zootecnia. 2008;37:1947-52.

5. Freyer G, Mayer M. Analysis of selected fecundity traits in two German pig breeds Landrasse and Edelschwein and their crossings. Züchtungskunde. 2012:84:500-10

6. Kovac M, Groeneveld E, Garcia-Cortez A. VCE 5.1.2. Computer Programme, FAL Neustadt/Mariensee. Germany. 2003.

7. Groeneveld E. PEST User's manual. FAL Neustadt/Mariensee, 1998.

8. Szulc K, Knecht D, Jankowska-Makosa A, Skrzypczak E, Nowaczewski S. The influence of fattening and slaughter traits on reproduction in polish large white sows. Italian J Anim Sci. 2013;12:e3.

9. Sell-Kubiak E, Duijvesteijn N, Lopes MS, Janss LLG, Knol EF, Bijma P, Mulder HA. Genome-wide association study reveals novel loci for litter size and its variability in a large white pig population. BMC Genomics. 2015;16(1049)

10. Argente MJ. Major Components in Limiting Litter Size, Insights from Animal Reproduction, Rita Payan Carreira (Ed.), InTech. 2016; https:/doi.org/10.5772/62280.

11. Distl O. Mechanisms of regulation of litter size in pigs on the genome level. Reprod Domestic Animal. 2007:42:10-6.

12. Muñoz M, Fernández Al, Olivo C, Munoz G, Rodriguez C, Fernández A, Alves E, Silió L. Non-additive effects of RBP4, ESR1 and IGF2 polymorphisms on litter size at different parities in a Chinese-European porcine line. Genet Sel Evol. 2010;42:23.

13. Dall'Olio S, Fontanesi L, Tognazzi L, Buttazzoni L, Gallo M, Russo V. ESR1 and ESR2 gene markers are not associated with number of piglets born alive in Italian large white sows. Italian J Anim Sci. 2011; https://doi.org/10.4081/ijas.2011.e35.

\section{Ready to submit your research? Choose BMC and benefit from:}

- fast, convenient online submission

- thorough peer review by experienced researchers in your field

- rapid publication on acceptance

- support for research data, including large and complex data types

- gold Open Access which fosters wider collaboration and increased citations

- maximum visibility for your research: over $100 \mathrm{M}$ website views per year

At BMC, research is always in progress.

Learn more biomedcentral.com/submissions 